\title{
Prediction of Natural Gas Consumption in Different Regions of China Using a Hybrid MVO-NNGBM Model
}

\author{
Xiaoyu Wang, ${ }^{1}$ Dongkun Luo, ${ }^{1}$ Jianye Liu, ${ }^{1}$ Wenhuan Wang, ${ }^{1}$ and Guixin Jie ${ }^{2}$ \\ ${ }^{1}$ School of Business Administration, China University of Petroleum-Beijing, 18 Fuxue Road, Changping District, Beijing 102249, China \\ ${ }^{2}$ CNPC Great Wall Drilling Company, Beijing 100101, China \\ Correspondence should be addressed to Dongkun Luo; ldk8511@hotmail.com
}

Received 14 June 2017; Revised 22 October 2017; Accepted 25 October 2017; Published 15 November 2017

Academic Editor: Yakov Strelniker

Copyright (c) 2017 Xiaoyu Wang et al. This is an open access article distributed under the Creative Commons Attribution License, which permits unrestricted use, distribution, and reproduction in any medium, provided the original work is properly cited.

\begin{abstract}
The accurate and reasonable prediction of natural gas consumption is significant for the government to formulate energy planning. To this end, we use the multiverse optimizer (MVO) algorithm to optimize the parameters of the Nash nonlinear grey Bernoulli model (NNGBM $(1,1)$ ) and propose a hybrid MVO-NNGBM model to predict the natural gas consumption in 30 regions of China. The results indicate that the prediction precision of the hybrid MVO-NNGBM model is better than that of other grey-based models. According to the forecast results, China's natural gas consumption will grow rapidly over the next five years and reach 354.1 billion cubic meters $(\mathrm{bcm})$ by 2020 . Moreover, the spatial distribution of natural gas consumption will shift from being supply oriented towards being demand driven and will be mainly concentrated in coastal and developed provinces.
\end{abstract}

\section{Introduction}

As a clean low-carbon energy, natural gas is a realistic choice for the sustainable development of China's energy supply. Thus, accelerating the development of the natural gas industry and increasing the proportion of natural gas in primary energy consumption are an effective method for resolving environmental constraints, improving air quality, and achieving low-carbon sustainable development in China. In recent years, China's natural gas consumption has rapidly increased, and the contradiction between domestic supply and demand has been prominent [1]. Because China's natural gas consumption has significant regional differences [2], pipeline construction should be prioritized in areas with rapidly increasing gas demands. Therefore, accurate forecasting of natural gas demand in different regions of China is highly important for policy making and planning [3].

In the literature, various methods and technologies have been used to predict natural gas consumption. The most commonly used methods include the following models: econometric, neural networks, and grey prediction. As a traditional forecasting tool, econometric models have been extensively used in natural gas consumption forecasting. Huntington
[4] investigated industrial natural gas consumption via the autoregressive distributed lag (ADL) method. Khan [5] studied the dynamics of natural gas consumption in Pakistan and predicted the natural gas demand from 2012 to 2020 via an ordinary least squares (OLS) model. Bianco et al. $[6,7]$ predicted natural gas consumption by using a linear regression model with logarithmic transformations and considered gross domestic product per capita (GDP per capita), gas prices, and heating degree days (HDD). Akpinar and Yumusak [8] used the Holt-Winters exponential smoothing method and the autoregressive integrated moving average (ARIMA) model to forecast natural gas demand. Taşpinar et al. [9] used a seasonal ARIMAX model to predict daily natural gas consumption.

In recent years, artificial neural network (ANN) models have been broadly used in energy prediction. Brown et al. $[10,11]$ developed a feedforward ANN model to predict daily gas consumption and compared it with a linear regression model. Demirel et al. [12] predicted natural gas consumption using neural networks and other time series methods. The comparison results showed that the ANN model with an error back-propagation algorithm gives a better forecasting accuracy than other models. Rodger [13] used a fuzzy nearest 
neighbor neural network model to forecast the natural gas demand to reduce energy costs. Yu and $\mathrm{Xu}$ [14] proposed an improved and modified GA-BP model to predict the shortterm gas load. Szoplik [15] predicted the gas demand in Szczecin using a multilayer perceptron model (MLP) and considered the calendar and weather factors.

The grey prediction model has the advantages of needing less data and having highly precise predictions, and it has been widely used in natural gas consumption forecasts. Xie and Li [16] predicted natural gas consumption based on the grey model optimized using the genetic algorithm. Wang et al. [17] and Boran [18] applied the rolling GM (1,1) model to predict natural gas consumption. Ma and Liu [19] developed a novel time-delayed polynomial grey prediction model (TDPGM $(1,1)$ ) and compared it with other common prediction models. The results demonstrate that the TDPGM $(1,1)$ model outperforms the other models. Zeng and Li [3] proposed a self-adapting intelligent grey prediction model that can automatically optimize model parameters. Kumar and Jain [20] applied the Grey-Markov model, the rolling GM $(1,1)$ model, and singular spectrum analysis (SSA) to predict natural gas consumption.

China is a country with distinct regional differences in terms of natural gas consumption, and few historical data are available in specific regions. Therefore, the grey forecasting model can be used to forecast natural gas consumption in each area. After the grey prediction model was proposed, many new models and methods were developed. Xie and Liu [21] developed a discrete grey model (DGM) to solve the instability of the traditional GM $(1,1)$ model. Wen and Huang [22] proposed a grey Verhulst model to address Ushaped data which was further developed by Dai and Li [23]. Akay and Atak [24] applied the grey prediction model with a rolling mechanism approach to predict the electricity demand in Turkey. Chen et al. [25] proposed a nonlinear grey Bernoulli model (NGBM) and applied it to forecast the foreign exchange rate. Chen et al. [26] improved the NGBM using the Nash equilibrium concept and established a Nash NGBM (NNGBM). The NNGBM model contains two parameters, the background value $p$ and the power $n$, which enhance the adjustability of the NGBM model. In addition, the grey model can also be associated with other models, such as the Grey-Markov [27-29], Grey-Taguchi [30-32], GreyFuzzy [30, 33, 34], and Grey-Fourier models [35-37].

To improve the accuracy of the grey prediction model, a number of methods have been proposed for optimizing the parameters of the grey prediction model. Lin et al. [38] and Zhao et al. [39] optimized the grey action quantity and the development coefficient of the GM (1,1) using the artificial fish swarm algorithm and the moth-flame optimization (MFO) algorithm, respectively. Zhou et al. [40] optimized the parameters of the NGBM model using particle swarm optimization (PSO). Zhao et al. [41] used the multiverse optimizer (MVO) [42] to optimize the parameters of the $\operatorname{DGM}(1,1)$ model, and the results demonstrate that the MVODGM $(1,1)$ model has a more excellent performance than the other optimized DGM $(1,1)$ models. The objective of this study is to optimize the two parameters of the NNGBM using the MVO approach and to apply this hybrid MVO-NNGBM model to predict natural gas consumption in 30 regions of China. The model was developed and tested using input data between 2010 and 2015 and compared with the GM $(1,1)$, the grey Verhulst model, and the NGBM $(1,1)$ model. The main contributions of this study can be summarized as follows: We propose a novel hybrid MVO-NNGBM model to forecast the consumption of natural gas in different regions of China.

The remainder of the paper is organized as follows. The next section analyzes the current natural gas consumption in China and the regional differences. Section 3 describes the data and methodology of this study. The results and discussions are presented in Section 4. The final section summarizes the main conclusions.

\section{Regional Distribution of Natural Gas Consumption in China}

China's total natural gas consumption has been continually increasing. According to China's National Bureau of Statistics [43], China's natural gas consumption grew to $194.7 \mathrm{bcm}$ in 2015, which represented an increase of $74.4 \%$ from that in 2010. In terms of sector classification, the natural gas end users are classified into different categories as follows: industrial, residential, transport, power, and heat use. In 2015, $51 \%$ of natural gas was used in the industrial sector, followed by residential (18\%), power and heat use (14\%), and transport (12\%). Another characteristic of China's natural gas consumption is the noticeable regional differences. According to the National Bureau of Statistics (the main objectives of this study are the provinces of mainland China, excluding Taiwan, Hong Kong, and Macao. Tibet is not included because of the lack of statistical data in the Tibetan region), China can be divided into five natural gas consumption areas that include East China, West China, North China, Northeast China, and South-Central China. Figure 1 shows the proportion of natural gas consumption in various regions of China in 2010 and 2015. The western region is the largest consumer of natural gas in 2015 at $31 \%$ of the total, followed by East China (26\%), North China (20\%), South-Central China (17\%), and Northeast China (6\%). Compared with 2010, the proportion of natural gas consumption in the western region has declined, while the consumption in other areas has risen or remained unchanged.

Figure 2 shows the natural gas consumption in each province. In sum, natural gas consumption increased in most areas but decreased in individual provinces, including Inner Mongolia, Jilin, and Sichuan. The top five provinces of natural gas consumption include Sichuan, Jiangsu, Beijing, Xinjiang, and Guangdong, which together account for $39.7 \%$ of the total. The smallest five provinces of natural gas consumption include Yunnan, Guangxi, Guizhou, Jiangxi, and Ningxia, which account for only $3.4 \%$ of the total. China's natural gas demand is mainly concentrated in the developed regions and natural gas producing areas. There are two main reasons for this phenomenon. On the one hand, China's natural gas resources are mainly distributed in the Tarim, Sichuan, and Ordos Basins, which represent $80 \%$ of the total natural gas reserves. In addition, the construction of China's natural gas pipeline is not perfect, which limits the scope of natural gas 


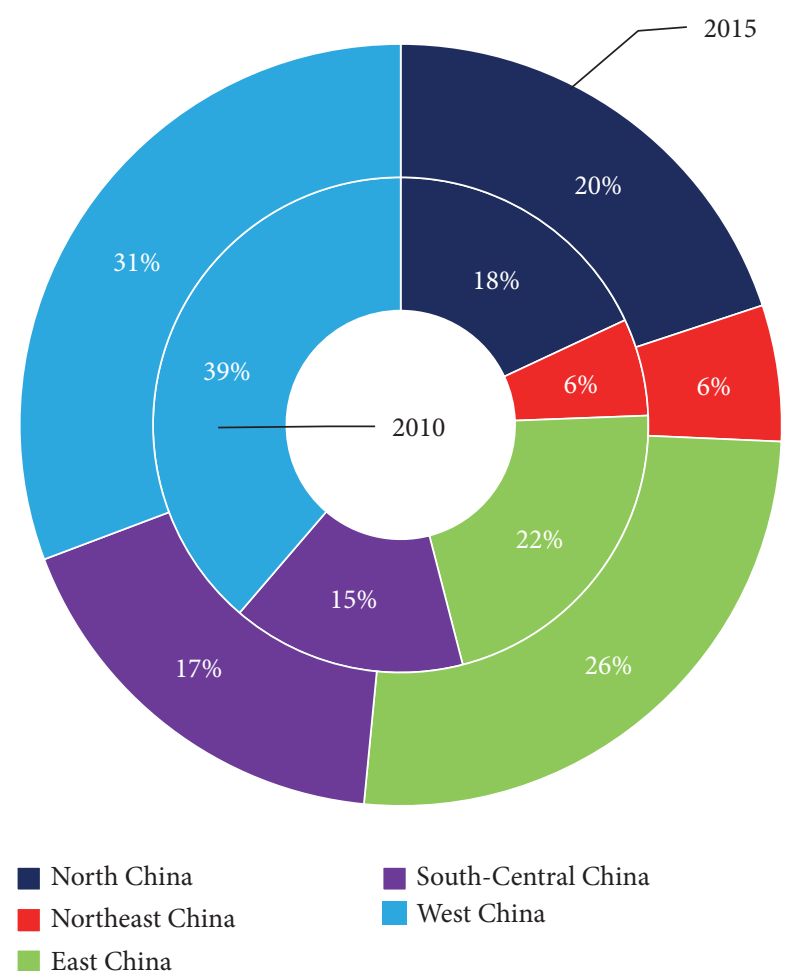

FIGURE 1: Proportion of natural gas consumption in various regions of China in 2010 and 2015.

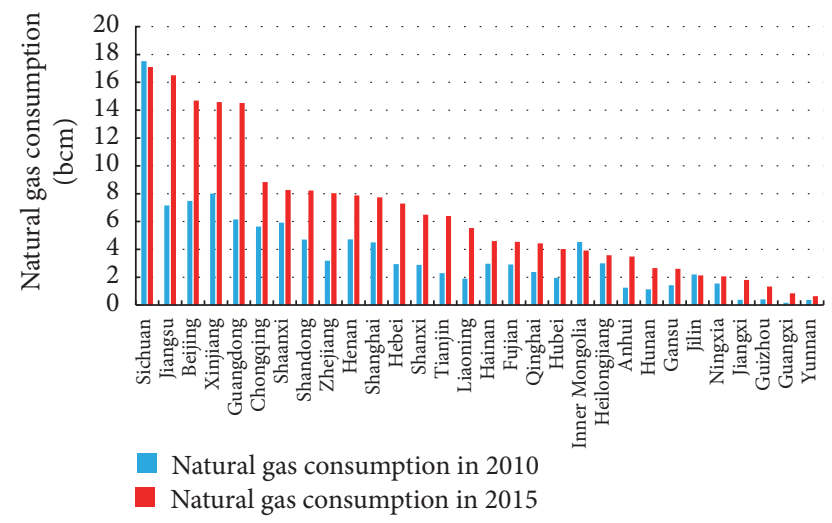

FIgURE 2: Natural gas consumption in China's 30 provinces in 2010 and 2015.

consumption. On the other hand, the population, economic environment, and industrial structure are different in different regions; thus, the demand for natural gas is also different.

\section{Data and Methods}

3.1. Data. In this paper, the dataset was obtained from the National Bureau of Statistics of China [43] on an annual basis from 2010 to 2015. Due to the lack of historical data in Tibet, Macao, Hong Kong, and Taiwan, this paper considers only the remaining 30 provinces as shown in Figure 2. In this study, we use the statistical data of natural gas consumption in 30 areas before 2013 as the modeling sample. To verify the model's predictive ability, the data from 2014 to 2015 will be used as the forecasted value for comparison with other models.

3.2. Nash NGBM (1,1) Model. Chen et al. [25] proposed the Nash NGBM, which is a modified GM $(1,1)$ combined with the Bernoulli differential equation. Compared with other grey-based models, the NNGBM model has better prediction performance to fit nonlinear data from small sample sizes. The key steps of the NNGBM $(1,1)$ are summarized as follows.

Step 1. Let $X^{(0)}$ be a nonnegative series

$$
X^{(0)}=\left\{x^{(0)}(1), x^{(0)}(2), \ldots, x^{(0)}(k), \ldots, x^{(0)}(m)\right\},
$$

where $x^{(0)}(k)$ is the $k$ th value of $x^{(0)}, k=1,2, \ldots, m$.

Step 2. Apply a one-time accumulated generating operation to construct $X^{(1)}$ :

$$
\begin{aligned}
& X^{(1)}=\left\{x^{(1)}(1), x^{(1)}(2), \ldots, x^{(1)}(k), \ldots, x^{(1)}(m)\right\}, \\
& x^{(1)}(k)=\sum_{i=1}^{k} x^{(0)}(i), \quad(k=1,2, \ldots, m) .
\end{aligned}
$$

Step 3. The grey differential equation of NGBM $(1,1)$ is defined as follows:

$$
x^{(0)}(k)+a z^{(1)}(k)=b\left(z^{(1)}(k)\right)^{r}, \quad k=2,3, \ldots, m,
$$

where $z^{(1)}(k)=P x^{(1)}(k)+(1-P) x^{(1)}(k-1), k=2,3, \ldots, m, P$ is the production coefficient of the background value, and $r$ is an adjustable parameter.

Step 4 . The parameters $a, b$ can be estimated by the least squares method:

$$
(a, b)^{T}=\left(B^{T} B\right)^{-1} B^{T} Y,
$$

where

$$
\begin{aligned}
& B=\left[\begin{array}{cc}
-z^{(1)}(2) & \left(z^{(1)}(2)\right)^{r} \\
-z^{(1)}(3) & \left(z^{(1)}(3)\right)^{r} \\
\vdots & \vdots \\
-z^{(1)}(m) & \left(z^{(1)}(m)\right)^{r}
\end{array}\right], \\
& Y=\left[\begin{array}{c}
x^{(0)}(2) \\
x^{(0)}(3) \\
\vdots \\
x^{(0)}(m)
\end{array}\right] .
\end{aligned}
$$

Step 5. The whitening differential equation of the $\operatorname{NGBM}(1,1)$ model is as follows:

$$
\frac{d x^{(1)}}{d t}+a x^{(1)}=b\left[x^{(1)}\right]^{r},
$$

where $a$ is the development coefficient and $b$ is the grey actor, $r \neq 1$. 
Step 6. Set the initial value $\widehat{x}^{(1)}(1)=x^{(0)}(1)$; thus, (6) can be expressed as follows:

$$
\begin{aligned}
& \hat{x}^{(1)}(k+1) \\
&=\left\{\frac{b}{a}+\left[\left(x^{(0)}(1)\right)^{1-r}-\frac{b}{a}\right] e^{-(1-r) a k}\right\}^{1 /(1-r)}, \\
& r \neq 1, k=1,2, \ldots, m .
\end{aligned}
$$

When $r=0$, (7) is the GM $(1,1)$ model; when $r=2$, (7) is the grey Verhulst model.

Step 7. The forecasted value of $\widehat{x}^{(0)}(k)$ can be estimated as follows:

$$
\begin{aligned}
& \widehat{x}^{(0)}(1)=x^{(0)}(1), \\
& \widehat{x}^{(0)}(k)=\widehat{x}^{(1)}(k)-\widehat{x}^{(1)}(k-1), \quad k=2,3, \ldots, m .
\end{aligned}
$$

The parameters of the NNGBM model include $a, b, P$, and $r$, which determine the accuracy of the predictive model. To obtain the best predictive value, the parameters must be optimized, where $a, b$ are determined by $P$ and $r$; therefore, only the two parameters $P$ and $r$ must be optimized.

3.3. Multiverse Optimizer Model. The MVO algorithm is proposed by Mirjalili et al. [44], and it is derived from the multiverse ideas in physics and builds mathematical models based on three main concepts of the multiverse theory: white holes, black holes, and worm holes. Suppose that each variable in the problem to be optimized is an object in the universe and follows the following rules in the optimization process:
(1) A high expansion rate has a high probability of a white hole and a low probability of a black hole.

(2) The universe with a higher expansion rate transmits objects through white holes, and the universe with a low expansion rate absorbs objects through black holes.

(3) All objects in the universe are unaffected by the expansion rate and move randomly through the wormhole towards the best universe.

The mathematical description of the MVO algorithm is as follows.

Assumptions.

$$
U=\left|\begin{array}{cccc}
x_{1}^{1} & x_{1}^{2} & \cdots & x_{1}^{d} \\
x_{2}^{1} & x_{2}^{2} & \cdots & x_{2}^{d} \\
\vdots & \vdots & \vdots & \vdots \\
x_{n}^{1} & x_{n}^{2} & \cdots & x_{n}^{d}
\end{array}\right|
$$

where $U$ is a randomly created universe, $d$ is the number of variables, and $n$ is the number of universes.

$$
x_{i}^{j}= \begin{cases}x_{k}^{j} & r 1<N I(U i) \\ x_{i}^{j} & r 1 \geq N I(U i),\end{cases}
$$

where $x_{i}^{j}$ is the $j$ th parameter of the $i$ th universe, $U i$ is the $i$ th universe, $N I$ is the expansion rate of the $i$ th universe, $r 1$ is a random number $\in[0,1]$, and $x_{k}^{j}$ is the $j$ th parameter of the $k$ th universe chosen based on the turntable mechanism.

To maintain the diversity and development capacity of the universe, it is believed that wormholes randomly transport objects in the universe and move towards the best universe. This mechanism is expressed as follows:

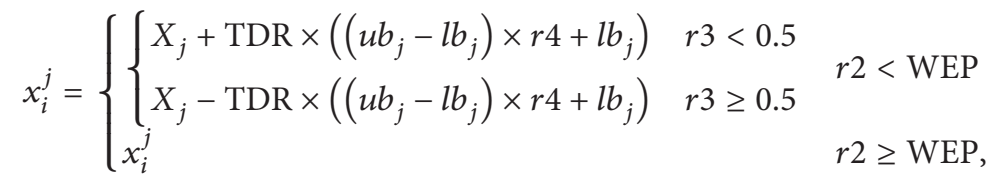

where $X_{j}$ is the $j$ th parameter of the best universe, WEP is the wormhole existence probability, TDR is the traveling distance rate, $u b_{j}$ and $l b_{j}$ are the upper and lower bounds of the $j$ th variable, and $r 2, r 3, r 4$ are random numbers in $\in[0,1]$.

The calculation formulas for WEP and TDR are as follows:

$$
\begin{aligned}
& \mathrm{WEP}=\min +l \cdot\left(\frac{\max -\min }{L}\right), \\
& \mathrm{TDR}=1-\frac{l^{1 / p}}{L^{1 / p}},
\end{aligned}
$$

where $\max (1$ in this paper) and $\min (0.2$ in this paper) are the maximum and minimum values of WEP, $l$ is the current iteration number, $L$ is the maximum number of iterations, and $p$ (6 in this paper) is the iterative precision in the development process. In the MVO model, Low WEP and High TDR underline exploration and local optima avoidance, while High WEP and Low TDR promote exploitation [41].

3.4. MVO-NNGBM (1,1) Model. In this subsection, we describe the processes applied to optimize the NNGBM parameters using the MVO algorithm. Figure 3 clearly shows the details of the hybrid MVO-NNGBM, and the implementation steps of the hybrid MVO-SVM algorithm are as follows.

Step 1. To weaken the randomness of the original time series, we use a log transformation to the original time sequence.

Step 2. Initialize the parameters. Before iterating, we should set some specific parameters that include the number of 


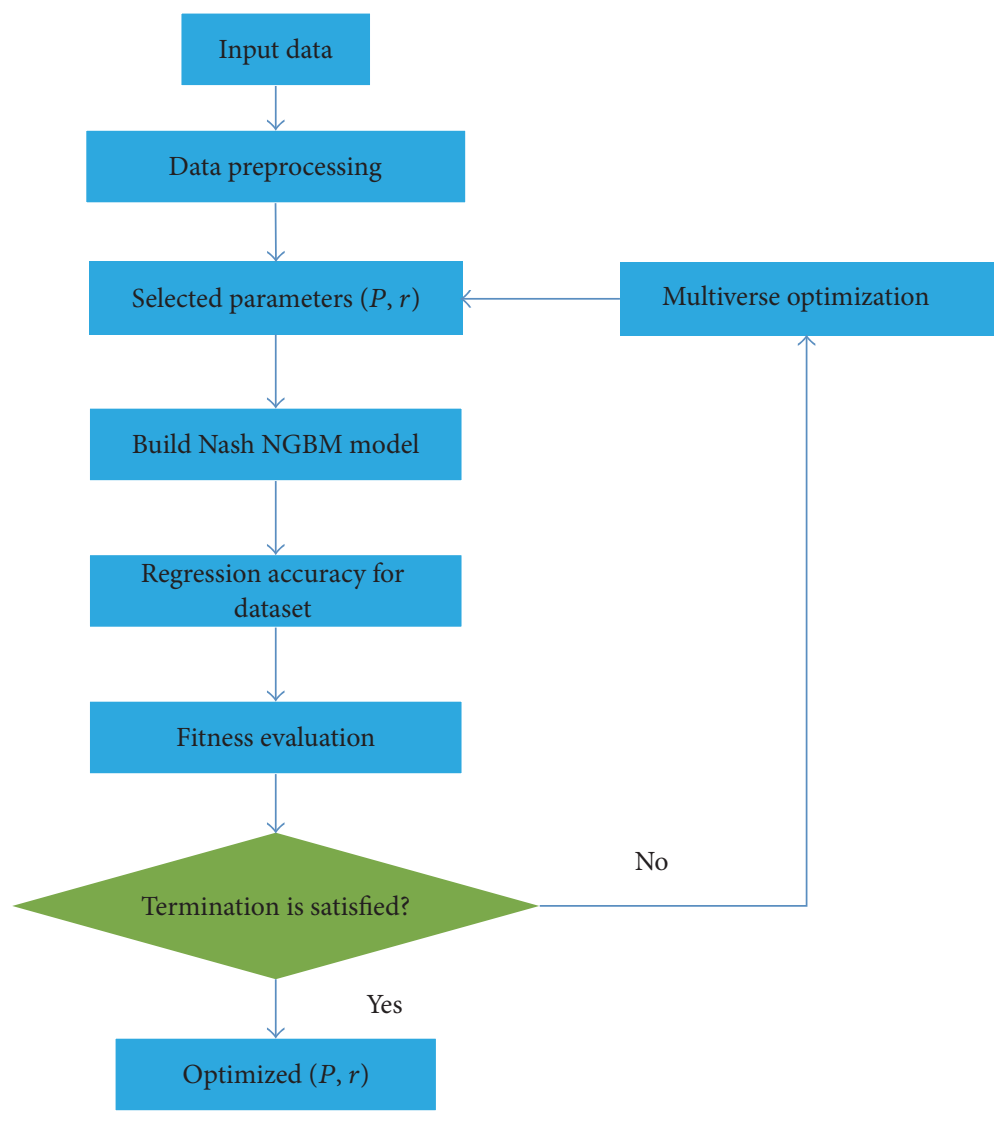

Figure 3: MVO-NNGBM flow chart.

universes $n$, the maximum number of iterations $T$, the number of variables $d$, upper bound $u b$, and lower bound $l b$.

In the MVO-NNGBM $(1,1)$ model, the two parameters $P$ and $r$ of NNGBM $(1,1)$ are substituted by the variables of universes $U$. First, we create random universes $U$ based on

$$
x_{0 j}=r *\left(u b_{j}-l b_{j}\right)+l b_{j},
$$

where $x_{0 j}$ is the $j$ th variable of matrix $U$ and $r$ indicates the random number produced by the uniform distribution between $[0,1]$.

Step 3. Determine the fitness function of the MVO algorithm. This article selects the mean squared error (MSE) as the fitness value, and the formula is as follows:

$$
\begin{array}{ll}
\min & \text { MSE }=\min \frac{1}{n-1} \sum_{i=1}^{n}\left(y_{i}-\widehat{y}_{i}\right)^{2} \\
\text { s.t } & P \in\left[P_{\min }, P_{\max }\right], \quad r \in\left[r_{\min }, r_{\max }\right],
\end{array}
$$

where $n$ is the sample number, $y_{i}$ is the observation value, and $\hat{y}_{i}$ is the predictive value.

Step 4. Calculate the fitness and seek the best universe.

In this section, we calculate the fitness values for all universes according to (15) and seek the best universe in each iteration. If the fitness function value of the $i$ th universe is less than that of the best universe found so far, the best universe and its fitness values will be updated until satisfying the end criterion. If the condition is satisfied, go to Step 5; otherwise, repeat Step 4.

Step 5. Output the best universe, that is, $P$ and $r$ and the corresponding fitness values. Then, input the optimal parameters $P$ and $r$ into the NNGBM $(1,1)$ model to forecast natural gas consumption.

We used the mean absolute percent error (MAPE) to measure the accuracy of the prediction models.

The types of MAPE are defined as follows:

$$
\mathrm{MAPE}=\frac{1}{n} \sum_{i=1}^{n}\left|\frac{y_{i}-\widehat{y}_{i}}{y_{i}}\right|,
$$

where $y_{i}$ is the actual value, $\hat{y}_{i}$ is the predicted value, and $n$ is the number of test sets.

A lower MAPE indicates higher accuracy. According to Lewis, MAPE values below 10\% indicate a higher prediction accuracy.

\section{Results and Discussion}

4.1. Results. In this part of the experiments, we use a novel hybrid MVO-NNGBM model to predict the natural gas 
TABLE 1: MAPE of prediction results for 30 regions in China.

\begin{tabular}{|c|c|c|c|c|c|}
\hline Region & MAPE Province & GM $(1,1)$ & Verhulst & $\operatorname{NGBM}(1,1)$ & MVO-NGBM \\
\hline \multirow{5}{*}{ North China } & Beijing & $16.0 \%$ & $2.9 \%$ & $5.9 \%$ & $3.1 \%$ \\
\hline & Tianjin & $21.8 \%$ & $2.4 \%$ & $6.3 \%$ & $2.8 \%$ \\
\hline & Hebei & $15.2 \%$ & $2.7 \%$ & $7.6 \%$ & $3.1 \%$ \\
\hline & Shanxi & $16.8 \%$ & $1.8 \%$ & $5.7 \%$ & $1.8 \%$ \\
\hline & Inner Mongolia & $4.8 \%$ & $5.7 \%$ & $4.6 \%$ & $4.6 \%$ \\
\hline \multirow{3}{*}{ Northeast China } & Liaoning & $20.8 \%$ & $12.9 \%$ & $9.2 \%$ & $6.2 \%$ \\
\hline & Jilin & $5.6 \%$ & $5.6 \%$ & $1.1 \%$ & $0.8 \%$ \\
\hline & Heilongjiang & $2.9 \%$ & $1.1 \%$ & $1.4 \%$ & $0.2 \%$ \\
\hline \multirow{7}{*}{ East China } & Shanghai & $6.3 \%$ & $1.3 \%$ & $1.4 \%$ & $1.3 \%$ \\
\hline & Jiangsu & $10.5 \%$ & $4.3 \%$ & $7.7 \%$ & $3.7 \%$ \\
\hline & Zhejiang & $14.6 \%$ & $5.0 \%$ & $6.7 \%$ & $4.9 \%$ \\
\hline & Anhui & $9.3 \%$ & $3.4 \%$ & $6.1 \%$ & $2.4 \%$ \\
\hline & Fujian & $7.4 \%$ & $6.2 \%$ & $7.0 \%$ & $5.8 \%$ \\
\hline & Jiangxi & $13.6 \%$ & $2.0 \%$ & $2.2 \%$ & $1.9 \%$ \\
\hline & Shandong & $8.5 \%$ & $2.4 \%$ & $2.4 \%$ & $2.3 \%$ \\
\hline \multirow{6}{*}{ South-Central China } & Henan & $8.1 \%$ & $4.6 \%$ & $3.8 \%$ & $2.1 \%$ \\
\hline & Hubei & $10.5 \%$ & $2.5 \%$ & $5.3 \%$ & $2.9 \%$ \\
\hline & Hunan & $10.2 \%$ & $2.0 \%$ & $3.3 \%$ & $1.4 \%$ \\
\hline & Guangdong & $4.1 \%$ & $5.1 \%$ & $6.1 \%$ & $0.2 \%$ \\
\hline & Guangxi & $10.8 \%$ & $7.9 \%$ & $8.3 \%$ & $9.3 \%$ \\
\hline & Hainan & $1.0 \%$ & $2.5 \%$ & $6.4 \%$ & $0.5 \%$ \\
\hline \multirow{9}{*}{ West China } & Chongqing & $7.5 \%$ & $1.5 \%$ & $2.1 \%$ & $1.5 \%$ \\
\hline & Sichuan & $3.7 \%$ & $5.8 \%$ & $1.8 \%$ & $1.4 \%$ \\
\hline & Guizhou & $32.4 \%$ & $5.4 \%$ & $6.8 \%$ & $5.0 \%$ \\
\hline & Yunnan & $9.0 \%$ & $57.8 \%$ & $14.9 \%$ & $3.9 \%$ \\
\hline & Shaanxi & $6.0 \%$ & $0.8 \%$ & $20.9 \%$ & $0.7 \%$ \\
\hline & Gansu & $10.3 \%$ & $3.1 \%$ & $5.1 \%$ & $0.2 \%$ \\
\hline & Qinghai & $5.7 \%$ & $2.3 \%$ & $4.8 \%$ & $2.5 \%$ \\
\hline & Ningxia & $4.0 \%$ & $3.9 \%$ & $10.9 \%$ & $3.8 \%$ \\
\hline & Xinjiang & $12.5 \%$ & $6.0 \%$ & $8.4 \%$ & $7.6 \%$ \\
\hline Average & & $10.3 \%$ & $5.7 \%$ & $6.1 \%$ & $2.9 \%$ \\
\hline
\end{tabular}

consumption of 30 regions in China from 2014 to 2015 and to compare the prediction performance with the GM $(1,1)$, grey Verhulst, and NGBM $(1,1)$ models. The four algorithms are evaluated using the datasets described earlier, and the results are listed in Table 1.

We use four prediction models to predict the consumption of natural gas in 30 provinces, and the forecast accuracy is shown in Table 1. Overall, the MAPE for the traditional GM $(1,1)$ model, grey Verhulst, $\operatorname{NGBM}(1,1)$, and hybrid MVO-NNGBM $(1,1)$ model is $10.3 \%, 5.7 \%, 6.1 \%$, and $2.9 \%$, respectively. From the individual point of view, the MAPE of 30 areas for the hybrid MVO-NNGBM $(1,1)$ model is less than $10 \%$, followed by the grey Verhulst model (28 areas), the NGBM $(1,1)$ model (27 areas), and the traditional GM $(1,1)$ model (16 areas). The results show that the hybrid MVO-NNGBM $(1,1)$ model exhibits much better prediction performance than all other models.

We further forecast the consumption of natural gas from 2016 to 2020, and the results are shown in Table 2. Overall, China's natural gas consumption is expected to grow to
$354.1 \mathrm{bcm}$ in 2020 , which represents an increase of $82 \%$ from the value in 2015. From an individual point of view, 19 provinces in China posted a positive growth rate in natural gas consumption, while the other regions posted a negative growth rate. The top five regions with the most substantial increase in natural gas consumption are Tianjin, Yunnan, Shanxi, Beijing, and Hebei. In contrast, the consumption of natural gas in Liaoning, Guangxi, Jilin, Henan, and Fujian will decline significantly. The spatial distribution of natural gas consumption in China in 2020 is shown in Figure 4. By 2020, China's natural gas consumption is mainly concentrated in North China, East China, Sichuan, Xinjiang, and Guangdong. The regional distribution of natural gas consumption shows distinct characteristics and is mainly concentrated in developed provinces and natural gas producing areas (see Figure 4).

4.2. Discussion. The results show that the hybrid MVONNGBM model can predict natural gas consumption more accurately than other grey prediction models mainly because 
TABLE 2: Predicted results of natural gas consumption in 30 regions of China $(\mathrm{bcm})$.

\begin{tabular}{|c|c|c|c|c|c|c|c|}
\hline Region & Province & 2016 & 2017 & 2018 & 2019 & 2020 & Growth rate \\
\hline \multirow{5}{*}{ North China } & Beijing & 17.7 & 22.1 & 27.9 & 35.7 & 46.3 & $215 \%$ \\
\hline & Tianjin & 8.6 & 12.2 & 17.9 & 27.2 & 43.2 & $575 \%$ \\
\hline & Hebei & 8.7 & 10.7 & 13.4 & 16.9 & 21.6 & $196 \%$ \\
\hline & Shanxi & 7.9 & 10.0 & 12.8 & 16.6 & 21.8 & $236 \%$ \\
\hline & Inner Mongolia & 4.0 & 3.9 & 3.8 & 3.7 & 3.6 & $-9 \%$ \\
\hline \multirow{3}{*}{ Northeast China } & Liaoning & 4.2 & 2.9 & 2.0 & 1.3 & 0.9 & $-83 \%$ \\
\hline & Jilin & 1.9 & 1.7 & 1.5 & 1.3 & 1.1 & $-47 \%$ \\
\hline & Heilongjiang & 3.6 & 3.6 & 3.5 & 3.5 & 3.4 & $-5 \%$ \\
\hline \multirow{7}{*}{ East China } & Shanghai & 7.8 & 7.8 & 7.7 & 7.6 & 7.5 & $-3 \%$ \\
\hline & Jiangsu & 18.6 & 21.7 & 25.6 & 30.3 & 36.1 & $119 \%$ \\
\hline & Zhejiang & 9.8 & 11.5 & 13.6 & 16.1 & 19.1 & $137 \%$ \\
\hline & Anhui & 3.8 & 4.1 & 4.3 & 4.4 & 4.6 & $32 \%$ \\
\hline & Fujian & 4.6 & 4.4 & 4.1 & 3.9 & 3.6 & $-20 \%$ \\
\hline & Jiangxi & 1.9 & 2.0 & 2.0 & 2.0 & 1.9 & $5 \%$ \\
\hline & Shandong & 8.6 & 8.9 & 9.2 & 9.5 & 9.8 & $19 \%$ \\
\hline \multirow{6}{*}{ South-Central China } & Henan & 7.1 & 6.5 & 5.8 & 5.1 & 4.5 & $-43 \%$ \\
\hline & Hubei & 4.5 & 4.9 & 5.2 & 5.6 & 5.9 & $48 \%$ \\
\hline & Hunan & 3.0 & 3.3 & 3.6 & 4.0 & 4.4 & $65 \%$ \\
\hline & Guangdong & 15.9 & 17.6 & 19.5 & 21.7 & 24.3 & $67 \%$ \\
\hline & Guangxi & 0.9 & 0.8 & 0.6 & 0.5 & 0.4 & $-54 \%$ \\
\hline & Hainan & 4.6 & 4.6 & 4.6 & 4.7 & 4.7 & $2 \%$ \\
\hline \multirow{9}{*}{ West China } & Chongqing & 9.6 & 10.4 & 11.3 & 12.3 & 13.4 & $52 \%$ \\
\hline & Sichuan & 18.6 & 20.2 & 22.2 & 24.6 & 27.3 & $60 \%$ \\
\hline & Guizhou & 1.7 & 2.0 & 2.5 & 3.0 & 3.5 & $161 \%$ \\
\hline & Yunnan & 0.8 & 1.1 & 1.6 & 2.4 & 4.1 & $549 \%$ \\
\hline & Shaanxi & 9.0 & 9.9 & 10.9 & 12.1 & 13.4 & $62 \%$ \\
\hline & Gansu & 2.6 & 2.6 & 2.5 & 2.4 & 2.3 & $-11 \%$ \\
\hline & Qinghai & 4.3 & 4.2 & 4.0 & 3.9 & 3.7 & $-16 \%$ \\
\hline & Ningxia & 2.0 & 2.0 & 2.0 & 1.9 & 1.9 & $-6 \%$ \\
\hline & Xinjiang & 16.1 & 16.3 & 16.3 & 16.1 & 15.8 & $8 \%$ \\
\hline Total & Average & 212.3 & 233.7 & 261.9 & 300.2 & 354.1 & $82 \%$ \\
\hline
\end{tabular}

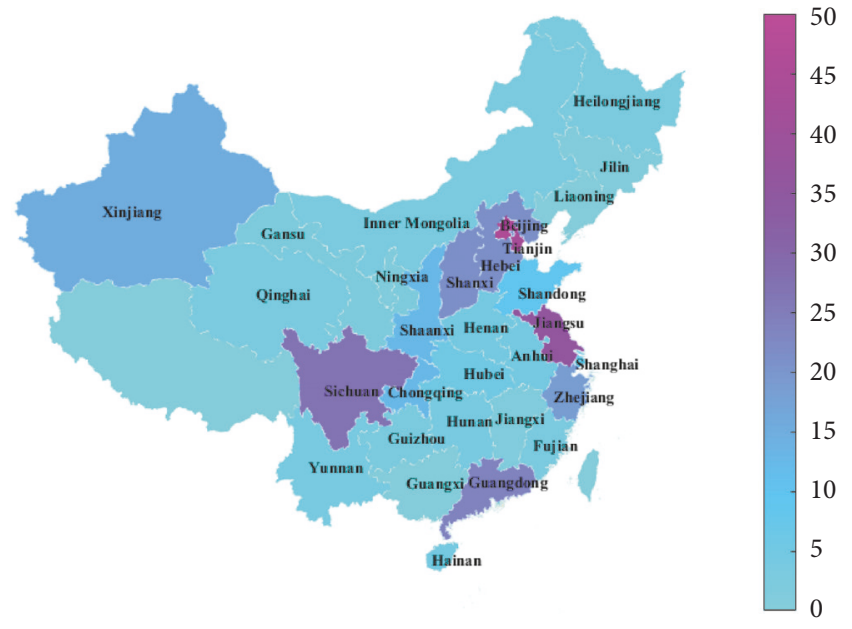

FIgURE 4: Natural gas consumption in China's provinces in 2020 $(\mathrm{bcm})$. Source: map generated by MATLAB using data from the prediction results of the Hybrid MVO-NNGBM. the MVO algorithm has a better global search ability. However, the prediction accuracy of the hybrid MVO-NNGBM model is not necessarily optimal for each province. The grey Verhulst model is more accurate than the MVONNGBM model for individual regions. We compare the MVO-NNGBM model with the available prediction models. Table 3 lists the accuracy of other prediction models. The comparison shows that the hybrid MVO-NNGBM model has the lowest MAPE for predicting natural gas consumption.

According to the forecast results, China's natural gas consumption is mainly concentrated in the coastal areas, Beijing, Tianjin, Hebei, Xinjiang, and Sichuan. The spatial distribution of natural gas consumption has shifted from being supply oriented towards being demand driven, which can be explained by three main reasons. First, China has accelerated the pace of pipeline construction to meet the needs of natural gas in coastal and developed provinces. According to the 13th Five-Year Plan's targets, natural gas pipeline mileage will reach 104 thousand $\mathrm{km}$ (64 thousand 
TABLE 3: Error comparisons with other methods.

\begin{tabular}{|c|c|c|c|}
\hline Source & Model & Target/region & MAPE \\
\hline \multirow{3}{*}{$\mathrm{Yu}$ and $\mathrm{Xu}[14]$} & Lm_MBP & \multirow{3}{*}{ Short-term natural gas load, China } & $5.00 \%$ \\
\hline & $\mathrm{Lm} \_\mathrm{ABP}$ & & $5.08 \%$ \\
\hline & $\mathrm{Lm} \_\mathrm{MABP}$ & & $5.15 \%$ \\
\hline \multirow{2}{*}{ Szoplik [15] } & MLP 22-25-1 & \multirow{2}{*}{ Monthly natural gas consumption, Szczecin (Poland) } & $5.75 \%$ \\
\hline & MLP 22-36-1 & & $8.23 \%$ \\
\hline \multirow{4}{*}{ Zhu et al. [45] } & FNF-SVRLP & \multirow{4}{*}{ Short-term gas demand, United Kingdom } & $3.80 \%$ \\
\hline & SVRLP & & $4.40 \%$ \\
\hline & ANN & & $5.30 \%$ \\
\hline & ARMA & & $6.10 \%$ \\
\hline Authors' & MVO-NNGBM & Natural gas consumption, China & $2.90 \%$ \\
\hline
\end{tabular}

$\mathrm{km}$ in 2015) by 2020, and the gas transmission capacity will increase from 280 billion cubic meters to 400 billion cubic meters. In the next five years, the Chinese government will accelerate the construction of gas pipelines to Beijing, Tianjin, and Hebei and increase the pipeline's capacity in North China. Moreover, natural gas pipelines along the Yangtze River economic belt will increase, and the gas supply capacity of the main trunk pipeline to the urban agglomerations in the middle Yangtze River will improve. Second, regional economic development in China is extremely uneven, which leads to differences in natural gas consumption. Provinces with higher economic development will have a higher demand for natural gas, especially in China's coastal areas. In contrast, natural gas demand in other less developed areas is growing slowly. Third, China's natural gas industry started late with a low degree of marketization. The regional gas market is less linked, which exacerbates regional differences in natural gas consumption. Based on the above reasons, the spatial distribution of natural gas consumption will shift from being supply oriented towards being demand driven, and regional differences will become more prominent.

\section{Conclusions}

With the rapid growth of natural gas consumption in China, accurate forecasting of natural gas consumption has become the focus of policy makers. Therefore, this paper uses the MVO algorithm to optimize the parameters of the NNGBM model and proposes a hybrid MVO-NNGBM model. We use the historical data of natural gas consumption in 30 areas before 2013 as the modeling sample and the data from 2014 to 2015 as the forecasted value for comparisons with other models. Finally, we use the hybrid MVO-NNGBM model to predict natural gas consumption in 30 provinces from 2016 to 2020. The main conclusions of this paper are as follows.

(1) The overall prediction results indicate that the proposed hybrid MVO-NNGBM model has better prediction accuracy than the other forecasting models. The prediction results of each province show that the grey Verhulst prediction model is better than the hybrid MVO-NGBM model for individual areas.

(2) China's natural gas consumption has significant regional differences and is mainly concentrated in Xinjiang,
Sichuan, and other natural gas generation areas as well as in North China, East China, coastal regions, and other developed provinces. With the improvement in China's natural gas pipeline construction, the future spatial distribution of natural gas consumption will shift from being supply oriented towards being demand oriented.

(3) The forecast results indicate that China's natural gas demand will continue to grow and is expected to achieve 354.1 bcm by 2020. Beijing, Tianjin, Zhejiang, Shanxi, and Hebei are the top five consumers of natural gas.

\section{Conflicts of Interest}

The authors declare that there are no conflicts of interest regarding the publication of this paper.

\section{Acknowledgments}

This research was supported by the National Science and Technology Major Project (2016ZX05042-002-004 and 2011ZX05018005-004) and the Science and Technology Special Projects of CNPC (2016D-4304).

\section{References}

[1] W. Lu, M. Su, B. D. Fath, M. Zhang, and Y. Hao, "A systematic method of evaluation of the Chinese natural gas supply security," Applied Energy, vol. 165, pp. 858-867, 2016.

[2] X. Li, "Natural gas in china - a regional analysis," 2015.

[3] B. Zeng and C. Li, "Forecasting the natural gas demand in China using a self-adapting intelligent grey model," Energy, vol. 112, pp. 810-825, 2016.

[4] H. G. Huntington, "Industrial natural gas consumption in the United States: An empirical model for evaluating future trends," Energy Economics, vol. 29, no. 4, pp. 743-759, 2007.

[5] M. A. Khan, "Modelling and forecasting the demand for natural gas in Pakistan," Renewable \& Sustainable Energy Reviews, vol. 49, article no. 4409, pp. 1145-1159, 2015.

[6] V. Bianco, F. Scarpa, and L. A. Tagliafico, "Analysis and future outlook of natural gas consumption in the Italian residential sector," Energy Conversion and Management, vol. 87, pp. 754764, 2014. 
[7] V. Bianco, F. Scarpa, and L. A. Tagliafico, "Scenario analysis of nonresidential natural gas consumption in Italy," Applied Energy, vol. 113, pp. 392-403, 2014.

[8] M. Akpinar and N. Yumusak, "Year ahead demand forecast of city natural gas using seasonal time series methods," Energies, vol. 9, no. 9, article no. 727, 2016.

[9] F. Taşpinar, N. Çelebi, and N. Tutkun, "Forecasting of daily natural gas consumption on regional basis in Turkey using various computational methods," Energy and Buildings, vol. 56, pp. 23-31, 2013.

[10] R. H. Brown, P. Kharouf, X. Feng, L. P. Piessens, and D. Nestor, "Development of feed-forward network models to predict gas consumption," in Proceedings of the 1994 IEEE International Conference on Neural Networks. Part 1 (of 7), pp. 802-805, June 1994.

[11] R. Brown and I. Matin, "Development of artificial neural network models to predict daily gas consumption," in Proceedings of the IECON '95 - 21st Annual Conference on IEEE Industrial Electronics, pp. 1389-1394, Orlando, FL, USA.

[12] Ö. F. Demirel, S. Zaim, A. Çališkan, and P. Özuyar, "Forecasting natural gas consumption in Istanbul using neural networks and multivariate time series methods," Turkish Journal of Electrical Engineering \& Computer Sciences, vol. 20, no. 5, pp. 695-711, 2012.

[13] J. A. Rodger, "A fuzzy nearest neighbor neural network statistical model for predicting demand for natural gas and energy cost savings in public buildings," Expert Systems with Applications, vol. 41, no. 4, pp. 1813-1829, 2014.

[14] F. Yu and X. Z. Xu, "A short-term load forecasting model of natural gas based on optimized genetic algorithm and improved BP neural network," Applied Energy, vol. 134, pp. 102-113, 2014.

[15] J. Szoplik, "Forecasting of natural gas consumption with artificial neural networks," Energy, vol. 85, pp. 208-220, 2015.

[16] Y. Xie and M. Li, "Research on prediction model of natural gas consumption based on grey modeling optimized by genetic algorithm," in Proceedings of the 2009 IITA International Conference on Control, Automation and Systems Engineering, CASE 2009, pp. 335-337, China, July 2009.

[17] J. Wang, H. Jiang, Q. Zhou, J. Wu, and S. Qin, "China's natural gas production and consumption analysis based on the multicycle Hubbert model and rolling Grey model," Renewable \& Sustainable Energy Reviews, vol. 53, pp. 1149-1167, 2016.

[18] F. E. Boran, "Forecasting natural gas consumption in Turkey using grey prediction," Energy Sources, Part B: Economics, Planning, and Policy, vol. 10, no. 2, pp. 208-213, 2015.

[19] X. Ma and Z. Liu, "Application of a novel time-delayed polynomial grey model to predict the natural gas consumption in china," Journal of Computational Applied Mathematics, 2017.

[20] U. Kumar and V. K. Jain, "Time series models (Grey-Markov, Grey Model with rolling mechanism and singular spectrum analysis) to forecast energy consumption in India," Energy, vol. 35, no. 4, pp. 1709-1716, 2010.

[21] N. Xie and S. Liu, "Research on Discrete Grey Model and Its Mechanism," in Proceedings of the 2005 IEEE International Conference on Systems, Man and Cybernetics, pp. 606-610, Waikoloa, HI, USA.

[22] K.-L. Wen and Y.-F. Huang, "The development of Grey Verhulst toolbox and the analysis of population saturation state in Taiwan-Fukien," in Proceedings of the IEEE International Conference on Systems, Man and Cybernetics (SMC '04), vol. 6, pp. 5007-5012, IEEE, October 2004.
[23] W. Dai and J. Li, "Grey Verhulst forward neural network model and its application," in Proceedings of the WCICA 2004 - Fifth World Congress on Intelligent Control and Automation, Conference Proceedings, pp. 204-206, chn, June 2004.

[24] D. Akay and M. Atak, "Grey prediction with rolling mechanism for electricity demand forecasting of Turkey," Energy, vol. 32, no. 9, pp. 1670-1675, 2007.

[25] C.-I. Chen, H. L. Chen, and S.-P. Chen, "Forecasting of foreign exchange rates of Taiwan's major trading partners by novel nonlinear Grey Bernoulli model NGBM $(1,1)$," Communications in Nonlinear Science and Numerical Simulation, vol. 13, no. 6, pp. 1194-1204, 2008.

[26] C.-I. Chen, P.-H. Hsin, and C.-S. Wu, "Forecasting Taiwan's major stock indices by the Nash nonlinear grey Bernoulli model," Expert Systems with Applications, vol. 37, no. 12, pp. 7557-7562, 2010.

[27] L.-C. Hsu, "Applying the Grey prediction model to the global integrated circuit industry," Technological Forecasting \& Social Change, vol. 70, no. 6, pp. 563-574, 2003.

[28] H. Samet and A. Mojallal, "Enhancement of electric arc furnace reactive power compensation using Grey-Markov prediction method," IET Generation, Transmission \& Distribution, vol. 8, no. 9, pp. 1626-1636, 2014.

[29] X. Huang and C. Tang, "Grey Markov model railway overload prediction," in Proceedings of the 2014 4th International Conference of Logistics Engineering and Management: System Planning, Supply Chain Management, and Safety, ICLEM 2014, pp. 349354, chn, October 2014.

[30] A. W. L. Yao and S. C. Chi, "Analysis and design of a TaguchiGrey based electricity demand predictor for energy management systems," Energy Conversion and Management, vol. 45, no. 7-8, pp. 1205-1217, 2004.

[31] M.-Y. Lin, C.-C. Tsao, C.-Y. Hsu, A.-H. Chiou, P.-C. Huang, and Y.-C. Lin, "Optimization of micro milling electrical discharge machining of Inconel 718 by Grey-Taguchi method," Transactions of Nonferrous Metals Society of China, vol. 23, no. 3, pp. 661-666, 2013.

[32] S. Roy, A. K. Das, and R. Banerjee, "Application of GreyTaguchi based multi-objective optimization strategy to calibrate the PM-NHC-BSFC trade-off characteristics of a CRDI assisted CNG dual-fuel engine," Journal of Natural Gas Science and Engineering, vol. 21, pp. 524-531, 2014.

[33] C. Zhou, Y. Cai, and Y. Wen, "Wind-speed prediction based on grey fuzzy mean generating function model," in Proceedings of the The 26th International Ocean and Polar Engineering Conference, International Society of Offshore and Polar Engineers, 2016.

[34] U. Munuswamy and T. P. Reddy, Multiobjective optimization of wedm process parameters on al5052/sic/gr hybrid mmc using grey fuzzy, 2016.

[35] L. I. Cun-Bin, L. I. Peng, and L. U. Gong-Shu, "Research of prediction of enterprise projects chain risk element transmission based on markov-fourier modified grey prediction model (mfgm)," Operations Research amd Management Science, vol. 22, pp. 241-247, 2013.

[36] Y.-T. Hsu, M.-C. Liu, J. Yeh, and H.-F. Hung, "Forecasting the turning time of stock market based on Markov-Fourier grey model," Expert Systems with Applications, vol. 36, no. 4, pp. 8597-8603, 2009.

[37] P. Delcea Camelia, Z. Wang, and L. Pei, "A Fourier residual modified Nash nonlinear grey Bernoulli model for forecasting 
the international trade of Chinese high-tech products," Grey Systems: Theory and Application, vol. 5, no. 2, pp. 165-177, 2015.

[38] Z.-S. Lin, Q.-S. Zhang, and H. Liu, "Parameter optimization of GM $(1,1)$ model based on artificial fish Swarm algorithm," in Proceedings of the IEEE International Conference on Grey Systems and Intelligent Services, pp. 166-177, 2011.

[39] H. Zhao, H. Zhao, and S. Guo, "Using GM (1,1) optimized by MFO with rolling mechanism to forecast the electricity consumption of inner mongolia," Applied Sciences (Switzerland), vol. 6, no. 1, pp. 1-18, 2016.

[40] J. Zhou, R. Fang, Y. Li, Y. Zhang, and B. Peng, "Parameter optimization of nonlinear grey Bernoulli model using particle swarm optimization," Applied Mathematics and Computation, vol. 207, no. 2, pp. 292-299, 2009.

[41] H. Zhao, X. Han, and S. Guo, "DGM $(1,1)$ model optimized by MVO (multi-verse optimizer) for annual peak load forecasting," Neural Computing and Applications, pp. 1-15, 2016.

[42] V. Martín and L. M. Robledo, "Multi-verse optimizer: A natureinspired algorithm for global optimization," Neural Computing and Applications, vol. 27, pp. 495-513, 2016.

[43] National Bureau of Statistics of China(NBSC), China statistics yearbook 2016, China Statistics Press, 2016.

[44] S. Mirjalili, S. M. Mirjalili, and A. Hatamlou, "Multi-verse optimizer: a nature-inspired algorithm for global optimization," Neural Computing and Applications, vol. 27, pp. 495-513, 2016.

[45] L. Zhu, M. S. Li, Q. H. Wu, and L. Jiang, "Short-term natural gas demand prediction based on support vector regression with false neighbours filtered," Energy, vol. 80, pp. 428-436, 2015. 


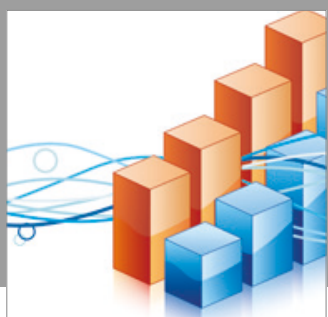

Advances in

Operations Research

vatersals

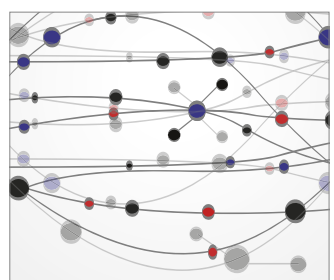

\section{The Scientific} World Journal
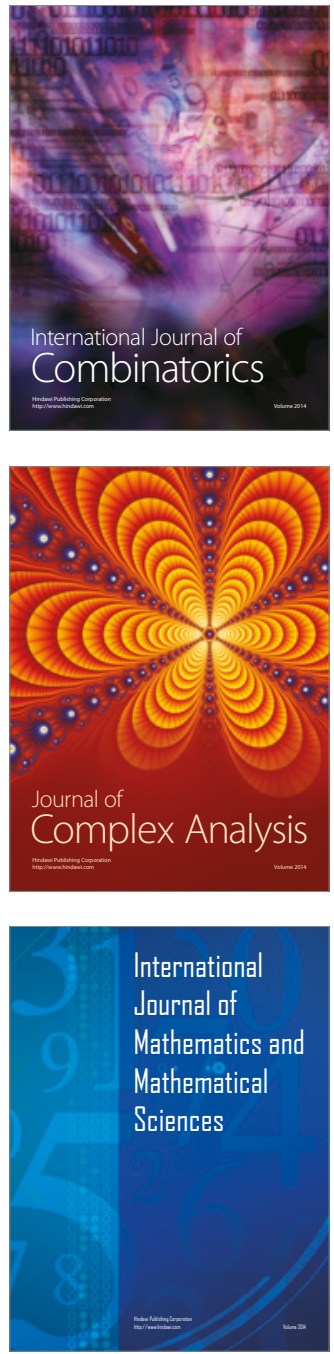
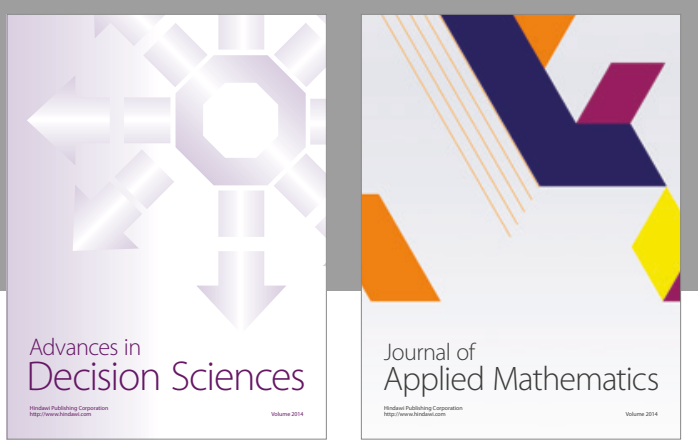

Algebra

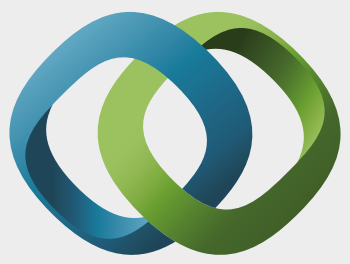

\section{Hindawi}

Submit your manuscripts at

https://www.hindawi.com
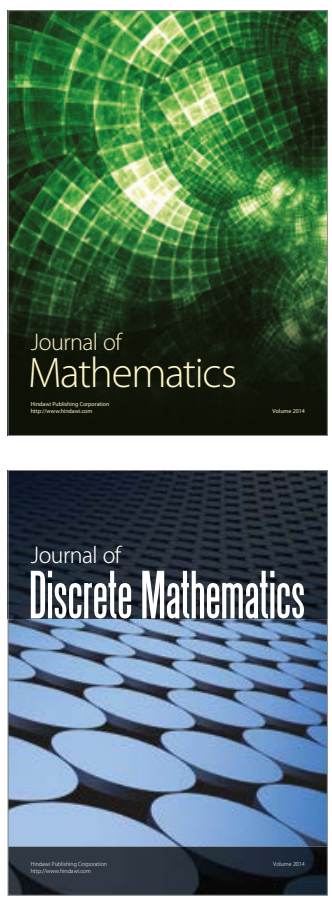

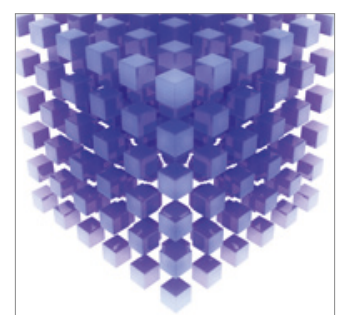

Mathematical Problems in Engineering
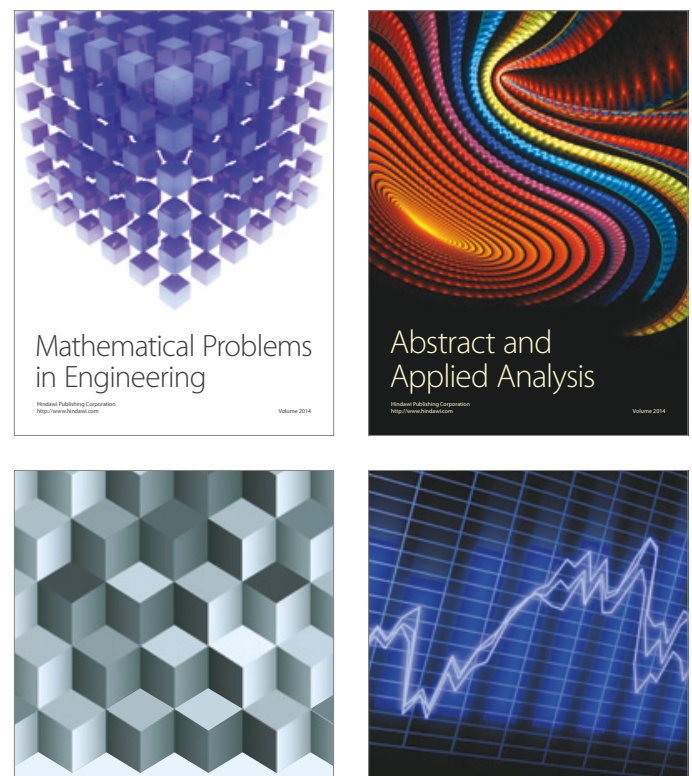

Journal of

Function Spaces

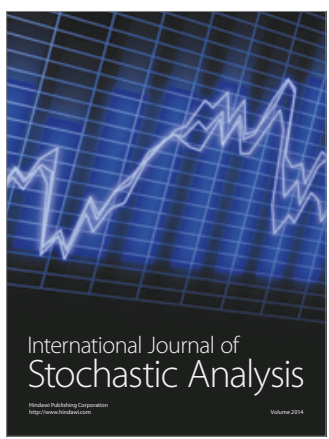

Probability and Statistics
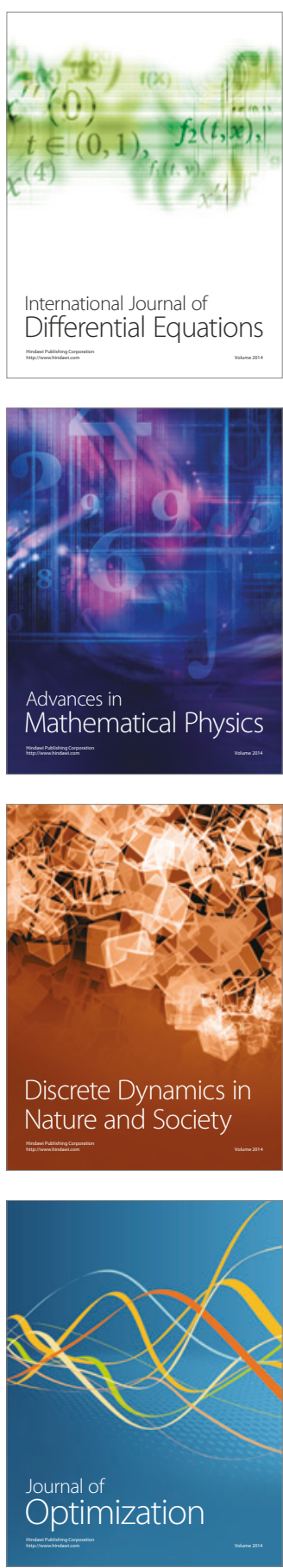